\title{
STUDY OF A VISUALIZATION SYSTEM OF THE CONCENTRATION DEGREE BY BIOLOGICAL SIGNALS -CASE OF ACTIVE LEARNING-
}

\author{
Kuniaki Yajima ${ }^{1}$, Keiichi Yonemura ${ }^{2}$, Yoshihiro Takeichi ${ }^{3}$ and Jun Sato ${ }^{3}$ \\ ${ }^{I}$ National Institute of Technology, Sendai College, Sendai, Miyagi, Japan \\ ${ }^{2}$ National Institute of Technology, Kisarazu College, Kisarazu, Chiba, Japan \\ ${ }^{3}$ National Institute of Technology, Tsuruoka College Tsuruoka, Yamagata, Japan
}

\begin{abstract}
Recently, Active Learning (AL) has been focused as the educational method. AL is different from traditional method of the class which is teacher-centric class, namely only teacher speak and students have the class passively [Ministry of Education, Culture, Sports, Science and Technology, 2012] [中部圏 23 大学 東海 A チーム 7 校（NUCB Undergraduate School.) , 2014]. AL is the style which students become active and they progress the class themselves actively. However, it is difficult to assess the students which have the AL, and it becomes hard about progressing the evolution of this style. The method of assesss this two perspectives. One is that teachers have been able to have the class well? The other one is that students quorily (knowledge and skill) has been able to progress well? Generally, assessment of class is questioneir-centric after closing the class at the end of semester. So we cannot feedback to our students that questionair's opinions. Moreover, generally the questionnaire to the students has included the issue that it is difficult to assess we want because subjectivity has strengthen. So we have advanced the investigation which can measure the degree of concentration against the class students have, using biological information as the assessment method we can measure it objectively. Using these results, we assess the degree of concentration against class of students while they are having the class of AL. This method makes us the situation which we can realize the visualization of the degree of actively while we realize the monitoring the situation of students in which they are having the class of AL. We use GSR, blinking, motion of the head as assessment indexes. We have selected the biological informations we measure mentioned above and equipment we will use in order to reduce the strain of students by wearing the equipment, although we can select and use a variety of biological informations and equipments to measure the situation of students. We can progress the class while visualizing the degree of concentration of students by using our system. As a result, we can reduce the class of AL which is imitative that is being a mere refrection of AL technique. Furthermore, we can apply our result to traditional teaching method because we can inform the situation which the students are becoming activated while the teachers are using a variety of skills which they drive the class well.
\end{abstract}

\section{KEYWORDS}

Active Learning, Eye Blinking, GSR, Evaluating System, Biological-Information

\section{INTRODUCTION}

Recently, measurement of electroencephalogram (EEG) and heart rate variability (HRA) are most general method as the assessment of biological information in the research of biopsychology. In addition, the index of biochemical substance is also used, for example, electro dermal activity (EDA), the temperature of skin surface, amount of hormonal secretion, and so on. Such indexes are called hemodynamic parameter; the assessment of these parameters has been advanced on the physiological research field. Although same research has been advanced on the field of e-learning and general education, they didn't have consensus because they have the different of the type physiological responses of students, and the different of the robustness. So it is the stage which researchers have been accumulated individualized approach each other so far. 
In the fiscal year of 2009, we studied a research of the physiological estimation using hemodynamic parameter as the physiology for educational material on the e-learning. In that research, we associated hemodynamic parameters with active coping against stress (pattern 1) and passive coping (pattern 2) behaviorally, as the typical patterns against psychological stress designed by experimental psychology. Pattern 1 appears by in literally active coping which they cope with a stress, that they can control it, themselves. Inversely, we can think that pattern 2 appears by passive coping which they wait out the stress while standing up the situation they have, for example, the noise is the situation that we don't have the method of efficient coping.

We prepared two different e-learning contents, and carried out the comparison experiment by measuring biological information based on hemodynamic parameter while students have the class with active learning. Two contents were as follows: one is that is dialog type content which is that lecture explains with video and students answer several simple questions while having a class, and the others is that is the non-dialog type content which has only explanations and that result with text. We measured the high frequency component (HF) as biological information, and measured systolic blood pressure (SBP), diastolic blood pressure (DBP), mean blood pressure (MBP), heart rate (HR), cardiac output $(\mathrm{CO})$, stoke volume (SV) and total peripheral resistance (TPR) as hemodynamic parameter. As the result of this experiment, we obtained the findings that a change of hemodynamic parameter evoked pattern 1, that is active coping against stress, while having the class with dialog-type content, and it also evoked pattern 2, that is passive coping, while having the class with non-dialog type content. Measurement result had the individual different on the studying time and the power of concentration by the influence of the degree of understanding about content, answer of questions, physical condition of student on the day they learned, and the time period of measurement. However, this result reported that we can identify the condition of having the class whether it is active or passive from student's blood current and the temperature of fingertip.

In the fiscal year of 2010, we focused on TPR which is a type of hemodynamic parameter. This is the result that measured a change of blood pressure on peripheral blood vessel of fingertip. As a result of this experiment, we observed the situation that blood pressure of students while having the class with contents changed with a cycle of from seven to eight minutes. Therefore, we collected the change of blood pressure of peripheral blood vessel by measuring the temperature of fingertip of students on real-time. As a result, they reported that body temperature decrease by decreasing of the power of concentration.

In the fiscal year of 2011, we measured the biological information using BIOPAC and Finometor, and researched causal relationship the power of concentration and biological information. we examined the result of previous research which we measured a variety of biological information, and decided to measure the degree of concentration of students by measuring the GSR, body temperature, and the pulse wave.

In the fiscal year of 2012, we carried out the measurement of effect of e-learning contents using biological information. The class advanced regardless of the degree of understanding of students on the e-learning, these contents of class is structuredness, it is difficult to keep the motivation of learning, and we can only assess situation of students using analysis after the class. So we developed the system which can assess the degree of concentration of student observing electrical skin resistance from forehead with real-time. The cause of emotional sweating is that is the sweating while exciting, and it evokes the decreasing of electrical skin resistance. As the result of that we measured the electrical skin resistance while on the concentration and non-concentration using measurement equipment we developed, we observed that GSR had low level by sweating while exiting, and reversely, electrical skin resistance had high level while cooling. This result suggested that we can identify the degree of concentration.

In the fiscal year of 2013, we suggested to assess by measuring GSR, body temperature, and beat in order to assess the degree of concentration of student while having an active learning class. We attempted increasing the accuracy of the identification of the degree of concentration by measuring additionally beat and body temperature in order to analysis the power of concentration while having the active learning class. We obtained the future task that need to be smaller of measurement system, and redesigned a part of measurement and redeveloped the system, because the students moved their body more on active learning class than while having the e-learning. We carried out the experiment using small measurement system we developed. Figure 1 and 2 has shown the experimental results. The result has shown the measurement on 20 minutes. We measured biological information with the cycle of five minutes. And students practiced the task for five minutes and had the rest for five minutes one after the other. As a result, GSR was changed in proportion to the degree of concentration while having active learning, and body temperature and beat had no change which we can identify to have the significant change. Clearly explain the nature of the problem, previous work, purpose, and contribution of the paper. 


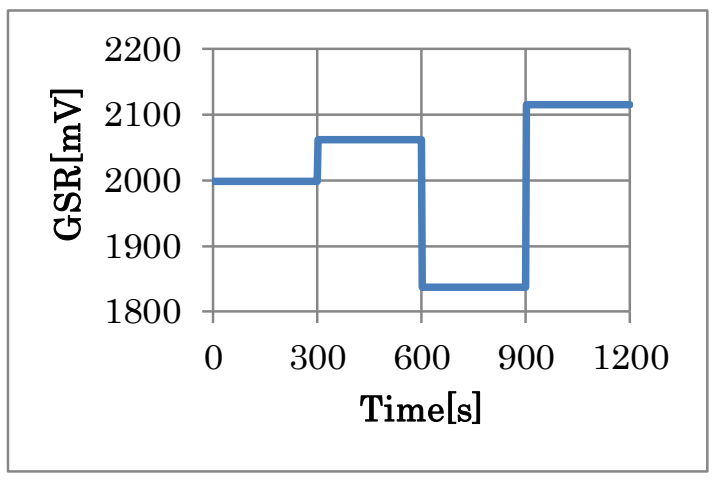

Figure 1. Average Value of GSR

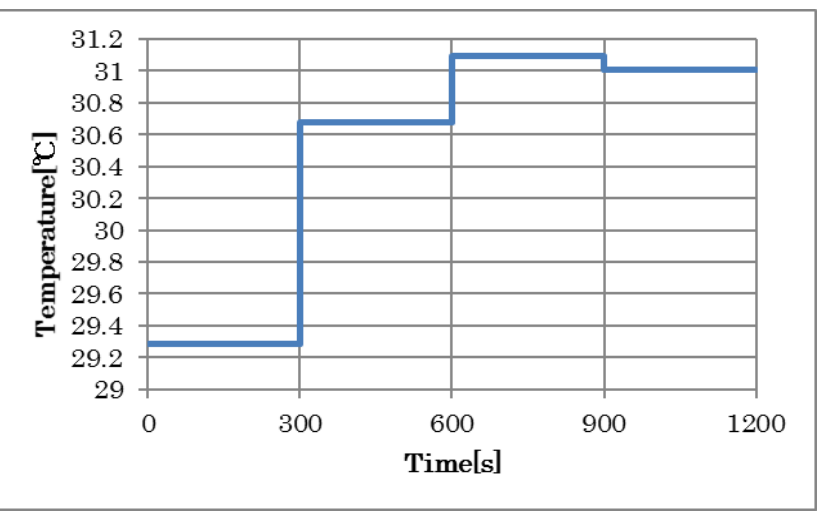

Figure 2. Average Value of Temperature

\section{EXPERIMENT}

The biological information is various information (for example, electrocardiogram, heart sound, X-ray absorption rate, etc.) emitted from the living body. In recent years, technology that utilizes such information has been applied in various fields such as medical care, nursing care, education. As an application example, there is also an approach called biofeedback that improves concentration by feeding back biological information to the visual and auditory sense.

Along with changes in human mental activity and state of consciousness, brain waves show different patterns. The brain wave at the time of awakening is an electroencephalogram that appears when consciousness concentration or stress is felt in a state of arousal, and it is called beta wave (13 to $20 \mathrm{~Hz}$ ). The brain wave at rest is an electroencephalogram that appears in a relaxed state or a resting closed eye state and is called an alpha wave ( 8 to $13 \mathrm{~Hz}$ ).

\subsection{Measuring Degree of Concentration by Eye Blinks}

People generally blink 15 to 20 times per minute. It is said that 3 to 4 physical blinks for moisturizing are enough and everything else is considered a psychoneurotic blink. Frequency of blinking of eyes decreases, such as when you are crazy, and tends to increase as the arousal level decreases. Frequency of blinking is greatly different between individuals and it is difficult to evaluate with absolute numbers, but mutations from normal times, relative numbers can be compared. Blinking can also be measured by changes in myoelectric potential [8]. For that reason, we adopt blinking as biometric information to use.

\subsection{Measuring Equipments}

In recent years, JIN CO., LTD., which develop the glasses brand "JINS", announce the glasses "JINS MEME" which is able to measure the fatigue degree of eye at work, the drowsiness during driving and the amount of activity. It has three types of sensors, three-point electrooculography sensors, three-axis accelerometer sensors and three-axis gyroscope sensors. Communication mode is Bluetooth Low Energy. JINS MEME can be obtain the data by connecting smartphone applications. We will verify the validity of relationship between concentration and biological-information by using the results, which are measured by the simple mearing instrument and a commercial wearable appliance such as JINS MEME.

In addition, in this research, in order to compare the change in the number of blinks and the brain wave, we use MindWave Mobile, a headset type electroencephalogram sensor developed independently by NeuroSky. JINS MEME has released SDK (Software Development Kit). SDK is provided for iOS and android, and in this research we developed using SDK for Android. SDK provides only real-time mode that can acquire data at about $20 \mathrm{~Hz}$. In order to carry out experiments, it is necessary to hold data, mainly to change the program of the output part so that outputted data can be output to CSR format on Android 
terminal's internal storage. Also added a method to output the timestamp at the same time as the acquired data. In addition, MindWave Mobile also provides SDK, and developed using that SDK. MindWave Mobile can acquire data obtained by removing noise from the acquired brain wave signal and converting it to a digital signal and data analyzed by the algorithm. MindWave mobile is able to output acquired data in CSV format to internal storage like JINS MEME.
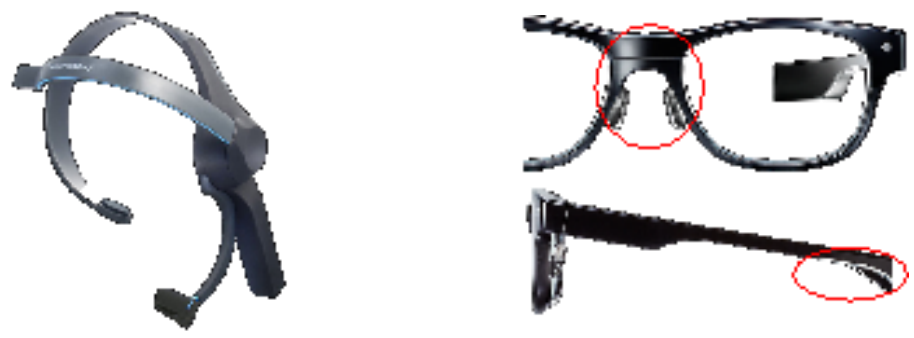

Figure 3. Measuring Devices

\section{RESULT}

Assuming actual AL, we conducted a discussion with three students. During the experiment, JINS MEME and Mindwave Mobile were worn by three of the students. Furthermore, in order to record the student's movements during the experiment, I recorded the situation of the experiment. As a content of the experiment, given a theme, three people talked about the subject. After that, after taking a break, we changed the person who wears the device again and carried out the experiment.

Experimental results of three subjects are shown in Figures 4-6. In addition, in order to investigate the relationship between the brain waves of individuals and the number of blinks, Table 1 shows correlation coefficients of each subject. Looking at the items related to blinks in the individual correlation, only the subject 2 has low correlation, but it turns out that there is almost no correlation between the other two persons. The reason for this result is that there are differences in time between brain waves and blinks depending on the degree of concentration and outliers in the number of blinks, and they do not consider it Can be considered. Therefore, we think that it is necessary to identify outliers and reexamine analysis methods.

Table 1. A Correlation Coefficient

\begin{tabular}{|c|c|c|c|}
\hline Test subject(1) & Alpha & Beta & Amonut of blinks \\
\hline Alpha & 1 & & \\
\hline Beta & 0.309708234 & 1 & \\
\hline Amonut of blinks & -0.184307091 & -0.177033061 & 1 \\
\hline Test subject (2) & Alpha & Beta & Amount of blinks \\
\hline Alpha & 1 & & \\
\hline Beta & 0.114314208 & 1 & \\
\hline Amount of blinks & -0.048206323 & 0.28841719 & 1 \\
\hline Test subject (3) & Alpha & Beta & Amount of blinks \\
\hline Alpha & 1 & & \\
\hline Beta & 0.357878084 & 1 & \\
\hline Amount of blinks & 0.105023702 & 0.078766294 & 1 \\
\hline
\end{tabular}




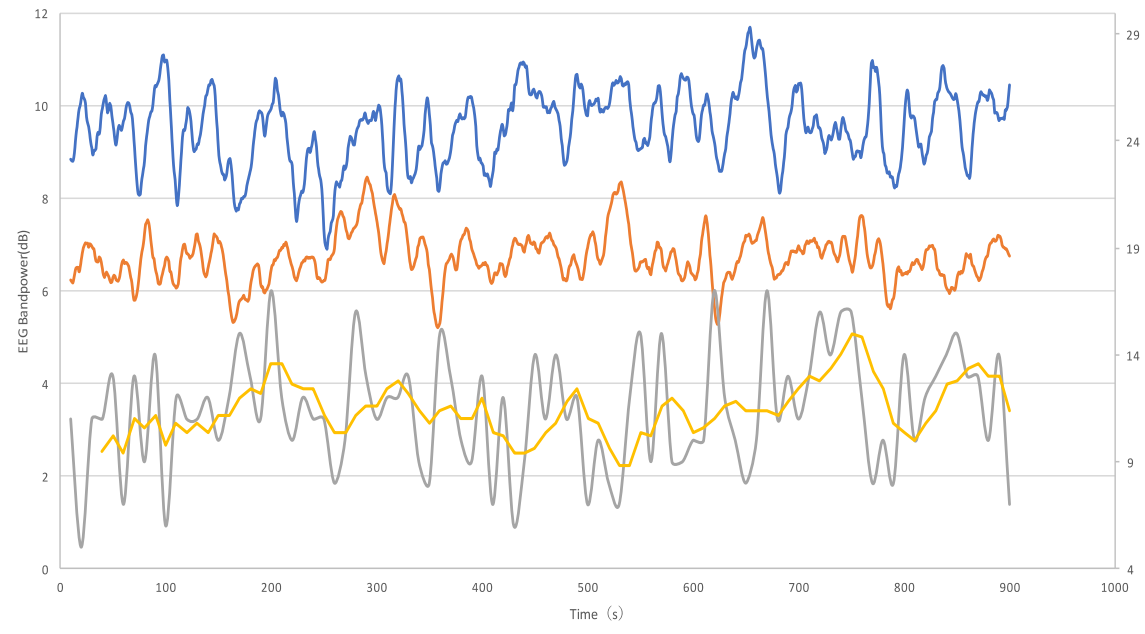

Figure 4. Results of Test Subjects 1

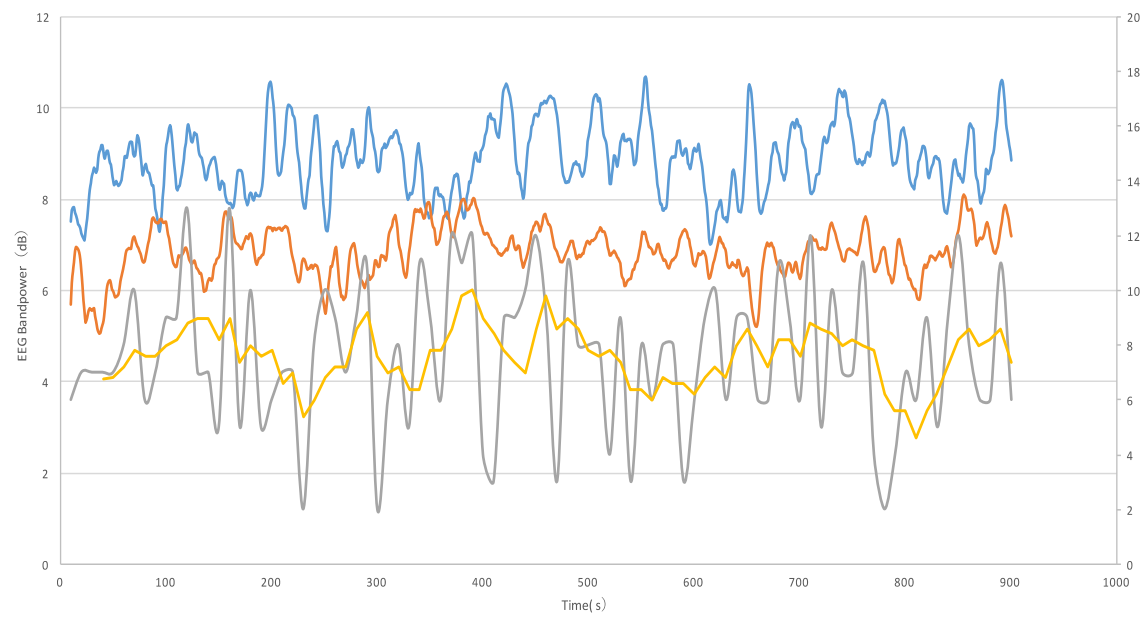

Figure 5. Results of Test Subjects 2

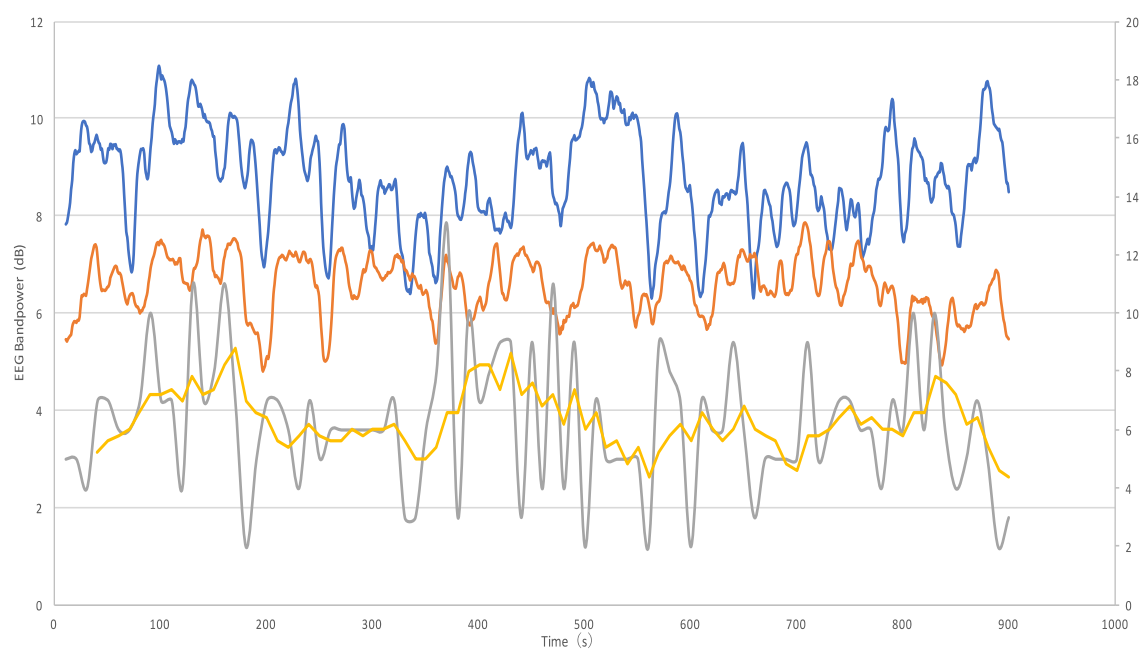

Figure 6. Results of Test Subjects 3 


\section{CONCLUSION}

In this paper, the power spectra of the alpha and beta waves of the students at the time of discussion and the number of blinks were compared and analyzed. As a result of the experiment, almost no correlation was found between blinking and brain waves. However, since each person has different characteristics in the brain wave information and the state of blinking, it is impossible to definitely declare significance with significance only by this result. In the future, in order to verify the significance of the data of this research, it is necessary to conduct additional experiments with an increased number of subjects and to reexamine the experimental method including the method of data analysis.

\section{ACKNOWLEDGEMENT}

This work was supported by JSPS KAKENHI Grant Number m16K00436.

\section{REFERENCES}

Ministry of Education, Culture, Sports, Science and Technology, 2012. Toward the qualitative change of university education to build a new future, Ministry of Education, Culture, Sports, Science and Technology, Japan, pp. 1-30. http.//www.mext.go.jp/b_menu/shingi/chukyo/chukyo0/toushin/1325047.html

Ministry of Education. Culture. Sports. Science and Technology. Active learning failed case handbook. Ministry of Education. Culture. Sports. Science and Technology, Japan, pp. 1-27, https.//www.nucba.ac.jp/archives/151/201507/ALshippaiJireiHandBook.pdf

O. Tetsuya et al, 2007. Evaluation of Doze Detection Criterion by Blink Obtained by the Correlation Analysis with EEG and SPL. IEICE Technical Report, MBE, Vol.107, No 72, p. 17-20

S. Tomono, 2013. With the "active learning" which connects with "deep learning.career guidance, Recrult Maketing Parthers Co. Ltd, Japan, pp. 2-4.

S. Watanabe, M.Shishido, 2016. Comparison of improving e ect of concentration in biofeedback of visual and auditory, society for science and technology, Vol 5, No 1, pp. 41-46.

Y. Adachi, 2013. Analysis of stress due to various stimuli by a variety of different ways, Journal of information science, Japan, pp. $65-76$.

N. Isao,B. Sadanao,2010. Person Authentication Using Brain Wave: Examination of Eye-Blink, IEICE technical report. Circuits and systems (CS2009 73-136), pp. 245-p246

T. Oketani et al, 2007. Evaluation of Doze Detection Criterion by Blink Obtained by the Correlation Analysis with EE G and SPL, IEICE Technical Report. MBE.107 (72). pp. 17-20. 